\title{
A Parallel Build-Up Algorithm for Global Energy Minimizations of Molecular Clusters Using Effective Energy Simulated Annealing
}

\author{
Thomas Coleman ${ }^{\dagger}$ David Shalloway ${ }^{\ddagger}$ and Zhijun $\mathrm{Wu}^{\S}$
}

\begin{abstract}
This work studies the build-up method for the global minimization problem for molecular conformation, especially protein folding. The problem is hard to solve for large molecules using general minimization approaches because of the enormous amount of required computation. We therefore propose a build-up process to systematically "construct" the optimal molecular structures. A prototype algorithm is designed using the anisotropic effective energy simulated annealing method at each build-up stage. The algorithm has been implemented on the Intel iPSC/860 parallel computer, and tested with the Lennard-Jones microcluster conformation problem. The experiments showed that the algorithm was effective for relatively large test problems, and also very suitable for massively parallel computation. In particular, for the 72-atom Lennard-Jones microcluster, the algorithm found a structure whose energy is lower than any others found in previous studies.
\end{abstract}

Abbreviated title: A Parallel Build-Up Algorithm for the Molecular Conformation Problem

Key words: global/local minimization, effective energy simulated annealing, parallel computation, protein folding

AMS (MOS) subject classification: 49M37,68Q22, 92C40

\footnotetext{
${ }^{\dagger}$ Department of Computer Science and Advanced Computing Research Institute, Cornell University, Ithaca, NY 14853.

${ }^{\ddagger}$ Section of Biochemistry, Molecular and Cell Biology, Cornell University, Ithaca, NY 14853.

${ }^{\S}$ Advanced Computing Research Institute, Cornell University, Ithaca, NY 14853.
} 


\section{Introduction}

We are interested in developing general build-up algorithms for the global minimization problem for molecular conformation, especially protein folding. The problem is important for biological studies [2]. But it is very difficult to solve because in theory even simple versions are $\mathcal{N} \mathcal{P}$-complete [7], and in practice the solution can hardly be made efficient because problem sizes are very large. Our model problem for this study is to find low energy structures of molecular clusters of identical atoms. Mathematically, the problem can be formulated as a global minimization problem for a nonlinear partially separable function. To be more specific, consider a molecular cluster of $n$ atoms, let $x=\left\{x_{i} \in \mathbf{R}^{3}, i=1, \ldots, n\right\}$ represent the molecular structure with each $x_{i}$ specifying the spatial position of atom $i$, and let the energy function be defined by $f(x)$,

$$
f(x)=\sum_{i=1, j>i}^{n} h\left(\left\|x_{i}-x_{j}\right\|\right),
$$

where $h$ is the pairwise energy function determined by the distance between each pair of atoms. Then the problem is

$$
\min _{x \in \mathbf{S}} f(x)
$$

where $\mathbf{S}$ is the set of all possible structures.

In recent years, the molecular conformation problem has been studied extensively in computational sciences, especially in the area of numerical optimization. Many approaches have been investigated, either deterministic or undeterministic, such as those in $[3,4,11,12,15,16,18,19,22]$. However, because of the enormous amount of required computation, most general minimization methods can only be applicable to small problem instances $(<100$ atoms), while problems of biological interests tend to be very large $(>1000$ atoms).

In this paper, we study a general scheme, the build-up method, for large molecular conformation problems. In general, the build-up method is to break up the molecule into its components and then rebuild it. Then the global energy minimization is conducted in two stages: the first for the components and the second for the molecule. The latter is started from a combination of the components that have the lowest energies in isolation. With 
this method, the solution of a large problem will hopefully become feasible, as the computation for each component should be more affordable (if not, the process can be applied recursively), and the second stage minimization may be made less expensive than started from an arbitrary structure.

The idea behind the build-up method is simple and straightforward, but whether or not it can be used effectively for large problems depends on many detailed factors such as the way of decomposing the molecule, the strategy for combining the components, and the method for the global minimization at each build-up stage. In this paper, we want to study the method and its related issues by considering the model problem in (2) and investigating a prototype build-up algorithm for it. Especially, we want to study how the global minimization procedure at each build-up stage can be designed efficiently given simple strategies for decomposing the molecule and combining the components.

In Section 2, we describe the prototype algorithm, its parallel implementation, and numerical experiments. The algorithm has been implemented on the Intel iPSC/860, and tested with the Lennard-Jones microcluster conformation problem. The experiments showed that the algorithm was effective for relatively large test problems, and also very suitable for massively parallel computation. In particular, for the 72-atom Lennard-Jones microcluster, the algorithm found a structure whose energy level is lower than any others found in previous studies. In Section 3, we describe the global minimization procedure used at each build-up stage. The procedure is designed specifically to take advantage of the build-up approach, and is a special anisotropic version of the effective energy simulated annealing algorithm proposed in [4]. Section 4 contains remarks on other related work and possible extensions of this study.

\section{Parallel Build-Up}

A simple build-up strategy has been taken for the prototype algorithm. The idea can be explained by considering the simple microcluster conformation problem. For a cluster of $n$ atoms, the algorithm assumes first that the lowest energy structure of an (n-1)-atom cluster has been found. Then the algorithm uses the optimized $(n-1)$-atom cluster together with a new atom to construct an initial $n$-atom structure, with which a global minimization procedure is 
started to minimize the energy of the $n$-atom cluster. Starting with such an initial structure, the procedure can hopefully find in a more effective way the lowest energy structure of the $n$-atom cluster.

To obtain the initial $n$-atom structure, the new atom is placed somewhere around the optimal structure of the $(n-1)$-atom cluster. Different positions for the new atom make different initial $n$-atom structures, which may lead to different conformation solutions. In our prototype algorithm, we make $n-1$ trials, each taking the initial structure with the new atom nearby one of atoms in the $(n-1)$-atom cluster. Eventually, the lowest energy structure found in these trials is taken as the candidate for the best structure of the $n$-atom cluster. Note that $n-1$ trials involve $n-1$ global minimizations which might need a great amount of computation. However, many trials could be avoided in practice by using problem specific heuristics. In any event, the minimizations are totally independent, and can be conducted in parallel with a very good efficiency. So the algorithm is naturally a parallel algorithm, and should also be suitable for massively parallel computation.

The above process can be generalized to a molecule of $n$-components with each component containing more than one atoms. Here we suppose that energies of components are minimized first so that each of them assumes a lowest energy structure.

Figure 1 contains an outline of the prototype build-up process, where the simplest decomposition is assumed, i.e., the molecule always is decomposed into $n$ components with each containing only one atom. Notation $N_{\epsilon}(x)$ for a 3-dimensional position $x$ defines a neighborhood of $x$. Formally, $N_{\epsilon}(x)=$ $\left\{y \in \mathbf{R}^{3},\|y-x\|<\epsilon, \epsilon>0\right\}$. Also, $x^{(k)}$ for some integer $k$ represents a 3-dimensional structure of a $k$-atom cluster, $x^{(k)}=\left\{x_{i}^{(k)} \in \mathbf{R}^{3}, i=1, \ldots, k\right\}$ where $x_{i}^{(k)}$ represents the 3 -dimensional position of atom $i$. Initial structures are constructed at Step 1 and 2. The energy of the cluster is minimized by the random search at Step 3. The method used for the random search is important for the entire build-up process. A general random search is basically not sensitive to initial structures, and hence can not take advantages of the build-up approach. A special random search thus is required to exploit the problem structures constructed by the build-up process so that short paths from initial structures to final solutions may be found. The motivation for pursuing the build-up approach is just to explore such an efficient search process. In next section, we discuss the anisotropic random search method 
used in our algorithm.

The build-up algorithm can be applied recursively to the (n-1)-atom (or $(n-1)$-component) cluster if its optimal structure is not available. In other words, the algorithm can be used systematically for small to large clusters so that solutions for larger and larger clusters can possibly be found. This process is similar to the polyhedral growth scheme in [9] for the LennardJones microcluster conformation, except that the latter exploited problem specific information, and conducted only local minimization at each growingup stage. The proposed build-up process here is more general and robust.

The build-up process is certainly not optimal for many problems. As a matter of fact, most problems might need more specific ways of decomposing the molecule and combining the components, e.g., special build-up procedures were used for some polypeptides in $[8,17]$. A better general approach to the build-up requires further research. However, this work is more concerned with how efficiently the global minimization, or the random search, at each build-up stage can be conducted given simple but general build-up strategies.

The parallel build-up algorithm in Figure 1 has been implemented on the Intel iPSC/860, and tested with the model problem in (2) using the Lennard-Jones pairwise potential function:

$$
h(\|y\|)=\frac{1}{\|y\|^{12}}-\frac{2}{\|y\|^{6}} .
$$

The function is widely used in molecular simulations, and provides a good model for the conformational energy that governs the behavior of particularly simple physical systems - molecular clusters of chemically inert atoms (e.g., argon). The conformation of this type of molecular clusters, also called the Lennard-Jones microcluster conformation, has been studied by generating polyhedral sequences [9], by taking simulated annealing method [22], by searching special lattice structures $[15,24]$, etc. These results provide standards for testing new algorithms.

In our previous study [4], the largest Lennard-Jones cluster whose optimal structure was found by a general global search process was the cluster of 54 atoms. So to test the build-up algorithm for relatively large problems, we start the test with the cluster of 55 atoms. Then larger clusters with up to 75 atoms are tested in turn based on the best solutions found for their predecessors. The results obtained for these clusters are listed in Table 1 
Algorithm 1 Parallel Build- $U p\}$

1 Input $x^{(n-1)^{*}} \quad$ \{optimal structure of (n-1)-atom cluster $\}$

2 For $i=1, \ldots, n-1$ do $\quad\left\{\right.$ initialize $\left.x^{(n)}\right\}$

$$
x_{i}^{(n)^{\circ}}=x_{i}^{(n-1)^{*}}
$$

3 For $i=1, \ldots, n-1$ do in parallel

$$
x_{n}^{(n)^{\circ}} \in N_{\epsilon}\left(x_{i}^{(n)^{\circ}}\right) \quad\{\text { add a new atom }\}
$$

Start random search with $x^{(n)^{\circ}}$

4 end

Figure 1: The parallel build-up algorithm. 
and compared with those obtained by the lattice search method in [15]. The results in [15] provide the best known solutions for most clusters with up to 147 atoms, which are used as standards for new findings. Note that in Table 1, "-" = the energy value in [15] was not obtained, "+" = the energy value in [15] was obtained, and " ++ " = the energy value is lower than that in $[15]$.

Our build-up algorithm found the best known energy values for most clusters in Table 1. Some values found by the algorithm were a bit higher than those by the lattice search method in [15]. However, for clusters of 66 and 72 atoms, the algorithm also found better solutions. The lower energy value for the cluster of 66 atoms was also discovered in [24] by relaxing the lattice search method in [15] and conducting more extensive searches. But the new energy value for the 72-atom cluster was lower than any others found before.

Table 2 illustrates further details about the performance of the algorithm for different initial structures. The energy values obtained by the algorithm for the 72-atom cluster are listed, each corresponding to a different initial structure. The initial structure was generated by placing a new atom nearby one of atoms in the optimized 71-atom cluster. The vector $x_{72}$ is the 3 dimensional position for the 72 th atom, the new one. For an integer $i, 1 \leq$ $i \leq 71, N_{\epsilon}\left(x_{i}\right)$ is an $\epsilon$-neighborhood of $x_{i}$, the position for the $i$ th atom in the optimized 71-atom cluster. As we can see in Table 2, several initial structures led the algorithm to the lowest energy structures of the 72 -atom cluster. Note that in Table 2, "-" = the best known energy value was not obtained, "+" = the best known energy value was obtained, and " $++"=$ the energy value is lower than the best known.

The program for the parallel build-up algorithm was written in standard C with some extensions for the Intel iPSC/860 interprocessor communications. No local linear algebra library routines were used. All results in Table 1 and Table 2 were obtained on the Intel iPSC/860 using 16 processors. However, the number of processors actually is scalable up to $n-1$ for an $n$-atom cluster problem. Figure 2 contains a performance example for the parallel build-up algorithm. The results were obtained by testing the algorithm with a 33-atom cluster problem on the Intel iPSC/860 using different number of processors. From these results we see that the algorithm achieved a very good parallel efficiency, and should be suitable for massively parallel computation. 


\begin{tabular}{|c|c|c|c|}
\hline \hline \multicolumn{5}{|c|}{ Low Energy Levels Found by LS and BU } \\
\hline \hline \# of atoms & ls & bu & remark \\
\hline 55 & $-2.79248 \mathrm{e}+02$ & $-2.792486 \mathrm{e}+02$ & + \\
\hline 56 & $-2.83643 \mathrm{e}+02$ & $-2.836438 \mathrm{e}+02$ & + \\
\hline 57 & $-2.88343 \mathrm{e}+02$ & $-2.883425 \mathrm{e}+02$ & + \\
\hline 58 & $-2.94378 \mathrm{e}+02$ & $-2.943784 \mathrm{e}+02$ & + \\
\hline 59 & $-2.99738 \mathrm{e}+02$ & $-2.996164 \mathrm{e}+02$ & - \\
\hline 60 & $-3.05876 \mathrm{e}+02$ & $-3.058756 \mathrm{e}+02$ & + \\
\hline 61 & $-3.12009 \mathrm{e}+02$ & $-3.120086 \mathrm{e}+02$ & + \\
\hline 62 & $-3.17354 \mathrm{e}+02$ & $-3.172686 \mathrm{e}+02$ & - \\
\hline 63 & $-3.23490 \mathrm{e}+02$ & $-3.234901 \mathrm{e}+02$ & + \\
\hline 64 & $-3.29620 \mathrm{e}+02$ & $-3.296205 \mathrm{e}+02$ & + \\
\hline 65 & $-3.34915 \mathrm{e}+02$ & $-3.349148 \mathrm{e}+02$ & + \\
\hline 66 & $-3.41043 \mathrm{e}+02$ & $-3.411108 \mathrm{e}+02$ & ++ \\
\hline 67 & $-3.47252 \mathrm{e}+02$ & $-3.472516 \mathrm{e}+02$ & + \\
\hline 68 & $-3.53395 \mathrm{e}+02$ & $-3.533774 \mathrm{e}+02$ & + \\
\hline 69 & $-3.59726 \mathrm{e}+02$ & $-3.597257 \mathrm{e}+02$ & + \\
\hline 70 & $-3.66892 \mathrm{e}+02$ & $-3.660450 \mathrm{e}+02$ & - \\
\hline 71 & $-3.73350 \mathrm{e}+02$ & $-3.733498 \mathrm{e}+02$ & + \\
\hline 72 & $-3.78524 \mathrm{e}+02$ & $-3.786371 \mathrm{e}+02$ & ++ \\
\hline 73 & $-3.84789 \mathrm{e}+02$ & $-3.839794 \mathrm{e}+02$ & - \\
\hline 74 & $-3.90909 \mathrm{e}+02$ & $-3.893122 \mathrm{e}+02$ & - \\
\hline 75 & $-3.96037 \mathrm{e}+02$ & $-3.957283 \mathrm{e}+02$ & - \\
\hline \hline
\end{tabular}

Table 1: Low energy values obtained for the Lennard-Jones microclusters of 55 to 75 atoms. Notation: $\mathbf{l s}$ - the lattice search method; bu - the parallel build-up algorithm. 


\begin{tabular}{|c|c|c|c|c|c|}
\hline \multicolumn{6}{|c|}{ Energy Values for the Cluster of 72 Atoms } \\
\hline$x_{72} \in$ & energy value & remark & $x_{72} \in$ & energy value & remark \\
\hline$N_{\epsilon}\left(x_{1}\right)$ & $-3.733492 \mathrm{e}+02$ & - & $N_{\epsilon}\left(x_{37}\right)$ & $-3.777450 \mathrm{e}+02$ & - \\
\hline$N_{\epsilon}\left(x_{2}\right)$ & $-3.764508 \mathrm{e}+02$ & - & $N_{\epsilon}\left(x_{38}\right)$ & $-3.384696 \mathrm{e}+02$ & - \\
\hline$N_{\epsilon}\left(x_{3}\right)$ & $-3.775487 \mathrm{e}+02$ & - & $N_{\epsilon}\left(x_{39}\right)$ & $-3.781465 \mathrm{e}+02$ & - \\
\hline$N_{\epsilon}\left(x_{4}\right)$ & $-3.781454 \mathrm{e}+02$ & - & $N_{\epsilon}\left(x_{40}\right)$ & $-3.502306 \mathrm{e}+02$ & - \\
\hline$N_{\epsilon}\left(x_{5}\right)$ & $-3.785242 \mathrm{e}+02$ & + & $N_{\epsilon}\left(x_{41}\right)$ & $-3.775498 \mathrm{e}+02$ & - \\
\hline$N_{\epsilon}\left(x_{6}\right)$ & $-3.785238 \mathrm{e}+02$ & + & $N_{\epsilon}\left(x_{42}\right)$ & $-3.774477 \mathrm{e}+02$ & - \\
\hline$N_{\epsilon}\left(x_{7}\right)$ & $-3.617191 \mathrm{e}+02$ & - & $N_{\epsilon}\left(x_{43}\right)$ & $-3.785232 \mathrm{e}+02$ & + \\
\hline$N_{\epsilon}\left(x_{8}\right)$ & $-3.786363 e+02$ & ++ & $N_{\epsilon}\left(x_{44}\right)$ & $-3.774939 \mathrm{e}+02$ & - \\
\hline$N_{\epsilon}\left(x_{9}\right)$ & $-3.786357 \mathrm{e}+02$ & ++ & $N_{\epsilon}\left(x_{45}\right)$ & $-3.781461 \mathrm{e}+02$ & - \\
\hline$N_{\epsilon}\left(x_{10}\right)$ & $-3.777808 \mathrm{e}+02$ & - & $N_{\epsilon}\left(x_{46}\right)$ & $-3.450495 \mathrm{e}+02$ & - \\
\hline$N_{\epsilon}\left(x_{11}\right)$ & $-3.771241 \mathrm{e}+02$ & - & $N_{\epsilon}\left(x_{47}\right)$ & $-3.660463 \mathrm{e}+02$ & - \\
\hline$N_{\epsilon}\left(x_{12}\right)$ & $-3.777386 \mathrm{e}+02$ & - & $N_{\epsilon}\left(x_{48}\right)$ & $-3.777437 \mathrm{e}+02$ & - \\
\hline$N_{\epsilon}\left(x_{13}\right)$ & $-3.786348 \mathrm{e}+02$ & ++ & $N_{\epsilon}\left(x_{49}\right)$ & $-3.203270 \mathrm{e}+02$ & - \\
\hline$N_{\epsilon}\left(x_{14}\right)$ & $-3.130972 \mathrm{e}+02$ & - & $N_{\epsilon}\left(x_{50}\right)$ & $-3.785237 \mathrm{e}+02$ & + \\
\hline$N_{\epsilon}\left(x_{15}\right)$ & $-3.781464 \mathrm{e}+02$ & - & $N_{\epsilon}\left(x_{51}\right)$ & $-3.777447 \mathrm{e}+02$ & - \\
\hline$N_{\epsilon}\left(x_{16}\right)$ & $-3.774205 \mathrm{e}+02$ & - & $N_{\epsilon}\left(x_{52}\right)$ & $-3.592840 \mathrm{e}+02$ & - \\
\hline$N_{\epsilon}\left(x_{17}\right)$ & $-3.774489 \mathrm{e}+02$ & - & $N_{\epsilon}\left(x_{53}\right)$ & $-3.785238 \mathrm{e}+02$ & + \\
\hline$N_{\epsilon}\left(x_{18}\right)$ & $-3.774916 \mathrm{e}+02$ & - & $N_{\epsilon}\left(x_{54}\right)$ & $-3.785241 \mathrm{e}+02$ & + \\
\hline$N_{\epsilon}\left(x_{19}\right)$ & $-3.660453 \mathrm{e}+02$ & - & $N_{\epsilon}\left(x_{55}\right)$ & $-3.785224 \mathrm{e}+02$ & + \\
\hline$N_{\epsilon}\left(x_{20}\right)$ & $-3.785221 \mathrm{e}+02$ & + & $N_{\epsilon}\left(x_{56}\right)$ & $-3.771202 \mathrm{e}+02$ & - \\
\hline$N_{\epsilon}\left(x_{21}\right)$ & $-3.774491 \mathrm{e}+02$ & - & $N_{\epsilon}\left(x_{57}\right)$ & $-3.786367 \mathrm{e}+02$ & ++ \\
\hline$N_{\epsilon}\left(x_{22}\right)$ & $-3.781450 \mathrm{e}+02$ & - & $N_{\epsilon}\left(x_{58}\right)$ & $-3.785231 \mathrm{e}+02$ & + \\
\hline$N_{\epsilon}\left(x_{23}\right)$ & $-3.777400 \mathrm{e}+02$ & - & $N_{\epsilon}\left(x_{59}\right)$ & $-3.785240 \mathrm{e}+02$ & + \\
\hline$N_{\epsilon}\left(x_{24}\right)$ & $-3.150841 \mathrm{e}+02$ & - & $N_{\epsilon}\left(x_{60}\right)$ & $-3.786363 \mathrm{e}+02$ & ++ \\
\hline$N_{\epsilon}\left(x_{25}\right)$ & $-3.723044 \mathrm{e}+02$ & - & $N_{\epsilon}\left(x_{61}\right)$ & $-3.771241 \mathrm{e}+02$ & - \\
\hline$N_{\epsilon}\left(x_{26}\right)$ & $-3.777809 \mathrm{e}+02$ & - & $N_{\epsilon}\left(x_{62}\right)$ & $-3.533947 \mathrm{e}+02$ & - \\
\hline$N_{\epsilon}\left(x_{27}\right)$ & $-3.587070 \mathrm{e}+02$ & - & $N_{\epsilon}\left(x_{63}\right)$ & $-3.785224 \mathrm{e}+02$ & + \\
\hline$N_{\epsilon}\left(x_{28}\right)$ & $-3.785232 \mathrm{e}+02$ & + & $N_{\epsilon}\left(x_{64}\right)$ & $-3.771230 \mathrm{e}+02$ & - \\
\hline$N_{\epsilon}\left(x_{29}\right)$ & $-3.411913 \mathrm{e}+02$ & - & $N_{\epsilon}\left(x_{65}\right)$ & $-3.615351 \mathrm{e}+02$ & - \\
\hline$N_{\epsilon}\left(x_{30}\right)$ & $-3.774303 \mathrm{e}+02$ & - & $N_{\epsilon}\left(x_{66}\right)$ & $-3.786363 \mathrm{e}+02$ & ++ \\
\hline$N_{\epsilon}\left(x_{31}\right)$ & $-3.777796 \mathrm{e}+02$ & - & $N_{\epsilon}\left(x_{67}\right)$ & $-3.774823 \mathrm{e}+02$ & - \\
\hline$N_{\epsilon}\left(x_{32}\right)$ & $-3.777807 \mathrm{e}+02$ & - & $N_{\epsilon}\left(x_{68}\right)$ & $-3.660451 \mathrm{e}+02$ & - \\
\hline$N_{\epsilon}\left(x_{33}\right)$ & $-3.785235 \mathrm{e}+02$ & + & $N_{\epsilon}\left(x_{69}\right)$ & $-3.786371 \mathrm{e}+02$ & ++ \\
\hline$N_{\epsilon}\left(x_{34}\right)$ & $-3.352482 \mathrm{e}+02$ & - & $N_{\epsilon}\left(x_{70}\right)$ & $-3.786367 \mathrm{e}+02$ & ++ \\
\hline$N_{\epsilon}\left(x_{35}\right)$ & $-3.774214 \mathrm{e}+02$ & - & $N_{\epsilon}\left(x_{71}\right)$ & $-3.785229 \mathrm{e}+02$ & + \\
\hline$N_{\epsilon}\left(x_{36}\right)$ & $-3.777800 \mathrm{e}+02$ & - & & & \\
\hline
\end{tabular}

Table 2: Energy values obtained with different initial structures. 


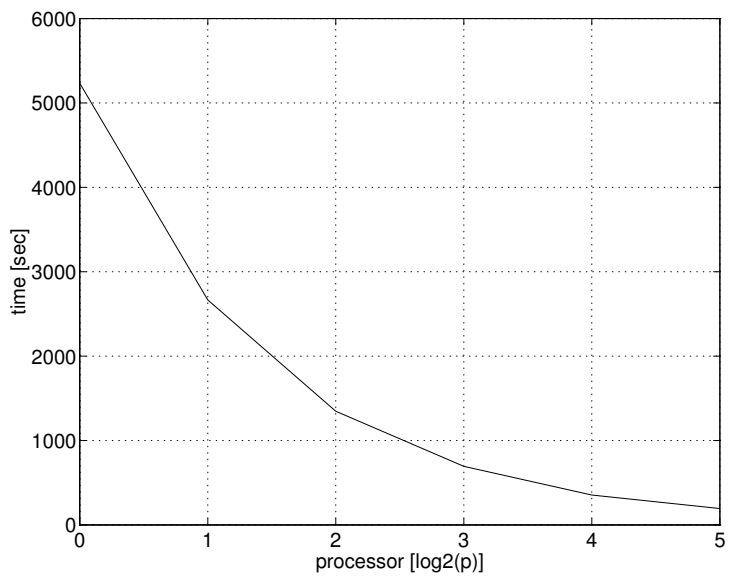

(a) timing

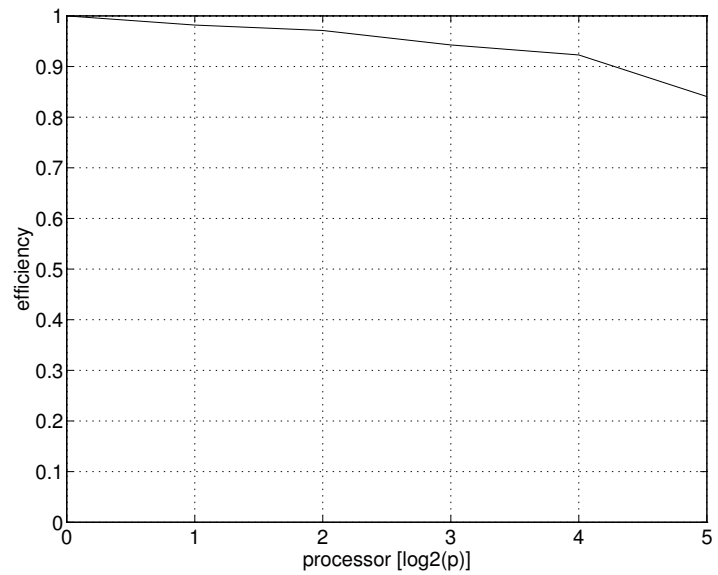

(b) parallel efficiency

Figure 2: A performance example for the parallel build-up algorithm. The results were obtained by testing the algorithm with a 33-atom cluster problem, for which up to 32 processors can be used. 


\section{Anisotropic Random Search}

In this section we describe the random search procedure used in Algorithm 1. The procedure is designed specifically to take advantages of initial structures constructed by the build-up process, so that short paths from these structures to the optimal solutions may possibly be found. Then hopefully, faster solutions can be obtained if better initial structures can be constructed. Here by "better" we mean "closer" to the optimal structure.

For a molecular cluster of $n$ atoms, the initial structure assumed by the build-up process in Algorithm 1 always contains a big component with $n-1$ atoms whose energy has been minimized in isolation. With the additional atom at a proper position, the structure can keep in low energy, and remain relatively stable. Hypothetically, such a structure may not be too far from the lowest energy structure, and the latter can possibly be obtained by making only small changes to the former. In addition, for the atoms in the $(n-1)$ atom component, those close to the additional atom may need relatively big changes in their positions - the closer, the bigger, and the farther, the smaller. For these reasons, we must have a special anisotropic random search procedure to simulate proper structural changes and then obtain efficient solutions.

We used for the random search a procedure called the anisotropic effective energy simulated annealing. The effective energy simulated annealing is a general random search method proposed in [4] for the energy minimization for molecular conformation. The method combines the simulated annealing with a class of effective energy functions transformed from the original energy function based on the theory of renormalization groups [13, 23]. For the details about the simulated annealing, the effective energy and the effective energy simulated annealing, readers are referred to $[1,10,22],[18,19]$ and $[4]$.

Figure 3 contains an outline for the effective energy simulated annealing algorithm, where $x$ is referred to as the current solution, $x_{+}$the perturbed solution, $\Delta x$ the random jump, $s$ the random step, $l_{k}$ the maximum number of random trials allowed at step $k, A_{k}$ the step size control at step $k, \Lambda$ and $T$ control parameters. Function random $[0,1)$ returns a random number in $[0,1)$.

In general, similar to simulated annealing, the effective energy simulated annealing generates a sequence of random trials with decreasing the param- 


$$
\begin{aligned}
& \text { Algorithm } 2 \text { Effective Energy Simulated Annealing }\} \\
& \begin{array}{l}
0\{\text { Initialization }\} \\
x=x_{\text {start }} \\
A_{1}=A_{\text {start }} \\
1\{\text { Iteration }\}
\end{array}
\end{aligned}
$$

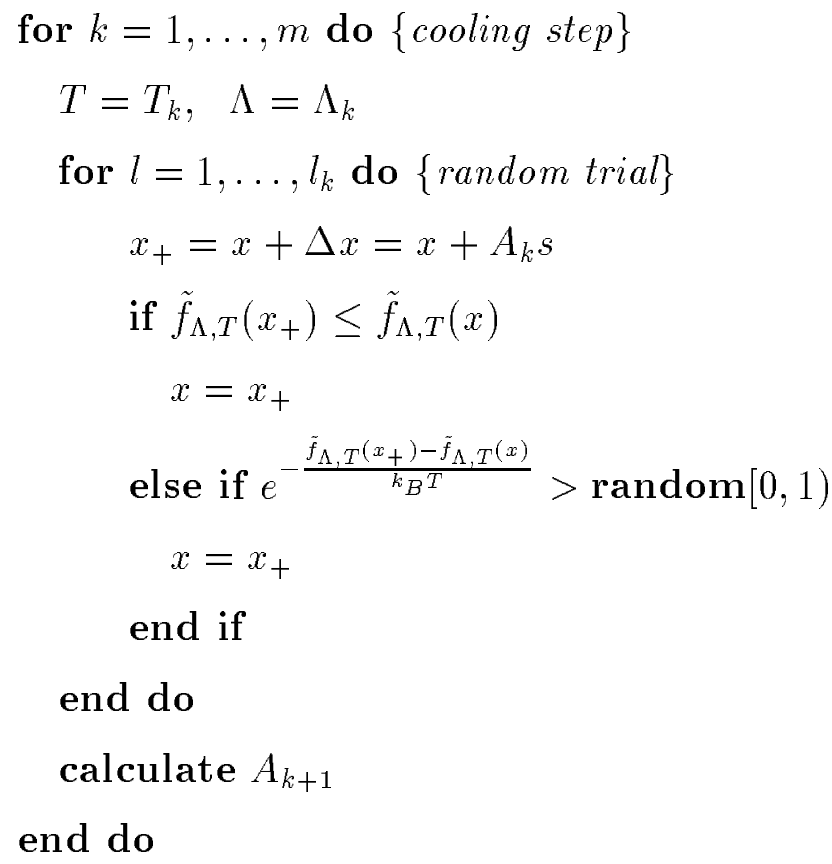

Figure 3: The effective energy simulated annealing. 
eter $T$ from positive to 0 . But in contrast to simulated annealing, the trials are not performed with the original energy function. Instead, for each $T$, the effective energy function $\tilde{f}_{\Lambda, T}$ defined as follows is used:

$$
\tilde{f}_{\Lambda, T}(x)=\sum_{i=1, j>i}^{n} \tilde{h}_{\Lambda_{i j}, T}\left(\left\|x_{i}-x_{j}\right\|\right),
$$

where $\Lambda$ is called a sampling parameter which can either be a scalar or a matrix. For the case of scalar, the subscript ij can be removed from $\Lambda_{i j}$. The function $\tilde{h}_{\lambda, T}(\|y\|)$ with respect to any $\lambda$ and $T$ is the pairwise effective energy function defined for given pairwise energy function $h(\|y\|)$ such that

$$
e^{-\frac{\tilde{h}_{\lambda, T}(\|y\|)}{k_{B} T}}=c_{\lambda} \int e^{-\frac{h\left(\left\|y^{\prime}\right\|\right)}{k_{B} T}} e^{-\left\|\left(y-y^{\prime}\right) / \lambda\right\|^{2}} d y^{\prime}
$$

where $k_{B}$ is the Boltzmann constant, and $c_{\lambda}$ is a normalization factor such that

$$
c_{\lambda} \int e^{-\left\|\left(y-y^{\prime}\right) / \lambda\right\|^{2}} d y^{\prime}=1
$$

Note that for any fixed $y$ and $T$,

$$
\lim _{\lambda \rightarrow 0} \tilde{h}_{\lambda, T}(\|y\|)=h(\|y\|) .
$$

Therefore, in the effective energy simulated annealing algorithm, the annealing process is applied to a sequence of effective energy functions obtained by changing values for $\Lambda$ from positive to 0 as $T \rightarrow 0$. The effective energy functions average in some sense the original energy variations over small regions, and therefore are somehow smoother functions. But as $\Lambda \rightarrow 0$, they become less smooth, and eventually converge to the original energy function.

In [4], an isotropic effective energy simulated annealing algorithm, a version of Algorithm 2 with $A_{k}$ and $\Lambda_{k}$ set to scalars, was implemented, and tested with a set of small molecular conformation problems. The results showed that the algorithm was more effective and efficient than a general simulated annealing method. However, the algorithm can not directly be used for the build-up process in Algorithm 1, simply because that the isotropic algorithm searches for solutions equally along all directions, which is not efficient, and not necessary, for the random search required by the buildup process. In terms of molecular conformation, the random search for the 
build-up should not make equal structural changes for all atoms, since otherwise, as we mentioned before, it will not be able to take advantage of the initial structures to obtain efficient solutions.

It suffices to construct an anisotropic version of Algorithm 2 that exploits special properties of the build-up process. To obtain such an algorithm, proper matrices for $A_{k}$ and $\Lambda_{k}$ need to be determined. In general, if $A_{k}$ can be determined, values for $\Lambda_{k}$ can be set proportional to, but smaller than, those for $A_{k}$. General approaches to the determination of $A_{k}$, as studied in $[20,21]$, typically require expensive computations. But for the buildup process, $A_{k}$ can simply be set to some values, as long as the resulting anisotropic random search makes special structural changes required by the process.

Algorithm 3 in Figure 4 describes the major strategies for computing $A_{k}$ and $\Lambda_{k}$ for the anisotropic version of Algorithm 2. In Algorithm 3, subscripts for $A_{k}$ and $\Lambda_{k}$ are removed since for all $k$ the same strategies apply. However $A_{k}$ and $\Lambda_{k}$ are proportional to $T_{k}$. So, values for $A_{k}$ and $\Lambda_{k}$ decrease as $T_{k}$ goes to 0 .

Using the strategies in Algorithm 3, $A$ is set to a diagonal matrix, whose $i$ th diagonal element $d_{i}$ is inversely proportional to the distance between atoms $i$ and $n$ in the initial structure (represented by $x^{\circ}$ ), where atom $n$ is the atom additional to the optimized $(n-1)$-atom component. With $A$ determined this way, the random search is forced to make large structural changes for the atoms closer to atom $n$ and small changes for others. For $\Lambda$, all its $i j$-elements are set to 0 if neither $i$ nor $j$ is equal to $n$, and all its $i n$-elements are set proportional to the step size $d_{i}$ for all $i<n$. The reason for this is that fluctuations between atoms $i$ and $j$ for $i<j<n$ are supposed to be small, and only those between atoms $i$ and $n$ for all $i<n$ need to be taken into account and sampled by the effective energy function.

In our implementation for the anisotropic random search, the cooling schedule, the number of random trials at each cooling step, the acceptance rate, etc. remained basically the same as the implementation for the isotropic effective energy simulated annealing in [4]. A local minimization also was applied at the very end of the search process. However, the starting temperature was set to a very low value to avoid large structural perturbations. Only 10 cooling steps were taken, and in total about $2^{5} n \log _{2} n$ function evaluations were required by the entire search. So for a cluster of 32 atoms, the total number of function evaluations for the anisotropic random search was about 


\section{Algorithm 3 \{Anisotropic Random Search\}}

$1 \quad\{$ Step Size Control $A\}$

$x_{+}=x+\Delta x$

$\Delta x=A s$

$A=\operatorname{diag}(d)$

$$
\begin{aligned}
& d_{i} \propto\left(1 /\left\|x_{n}^{\circ}-x_{i}^{\circ}\right\|\right)(T), \quad i=1, \ldots, n-1 \\
& d_{n}=\max _{1 \leq i<n}\left\{d_{i}\right\}
\end{aligned}
$$

$2 \quad\{$ Sampling Matrix $\Lambda\}$

$\Lambda_{i j}=0, \quad \forall i, j, \quad i<j<n$

$\Lambda_{i n} \propto d_{i}, \quad i=1, \ldots, n-1$

Figure 4: Anisotropic random search strategies. 
$5 \mathrm{~K}$, and for a cluster of 64 atoms, it was about $12 \mathrm{~K}$.

\section{Concluding Remarks}

We would like to point out that the build-up idea for molecular conformation is not new. It was introduced in $[8,17]$ for some polypeptide chain conformation problems. Basically, the methods in $[8,17]$ tried to first minimize the energies of smaller fragments, such as amino acids, and then combine the fragments to obtain optimal structures for larger units. The polyhedral growth scheme in [9] was also a sort of build-up approaches. The basic buildup strategy in this scheme - to construct the optimal structure for the cluster of $n$ atoms based on the optimal structure for the cluster of $n-1$ atoms - is also used by the prototype algorithm studied in this work.

Compared with $[8,17]$, our study on the build-up method is for a more general purpose - to find general and systematic build-up procedures and develop efficient random search methods specific to the build-up procedures. As we have mentioned before, the prototype algorithm developed in this study also is more general and robust than the polyhedral growth scheme.

We also want to mention that for the anisotropic random search, especially the anisotropic simulated annealing, a general approach based on some statistical methods was studied before in [21]. However this approach involves computing for the step size control a covariance matrix of random sampling at each cooling step. The computation is typically in $\mathcal{O}\left(n^{2} l\right)$, where $n$ is the problem dimension and $l$ the number of random trials made at one cooling step. For an anisotropic effective energy simulated annealing procedure this approach could be costly since $l$ is typically in $\mathcal{O}\left(n \log _{2} n\right)$.

In conclusion on this work, we studied the build-up method for molecular conformation, and proposed a prototype algorithm. By exploiting the special properties associated with the build-up process, an efficient random search procedure - anisotropic effective energy simulated annealing - was developed and used. The algorithm was tested with some relatively large microcluster conformation problems. The results were effective. Especially, better solutions were found by the algorithm for the 72-atom Lennard-Jones microcluster. The algorithm also proved easy to parallelize and suitable for massively parallel computation.

However, the build-up procedure in this study can only serve as a com- 
putational model. To extend the procedure to a variety of molecular conformation problems, e.g., the protein folding problem, still requires a lot more work. For example, in real applications, molecules may better be decomposed into many components with equal or not equal sizes. Then whether or not there is a general approach to combining components systematically and making efficient searches is not known. Also, the build-up method could be useful for solving a class of so-called "perturbed" protein folding problems finding the most stable structures for proteins of known structures with some small components added, deleted, or changed. Research along this direction would be interesting to pursue.

\section{Acknowledgements}

This research was supported partially by the Cornell Theory Center, which receives major funding from the National Science Foundation and IBM Corporation, with additional support from New York State and members of its Corporate Research Institute, and also supported, in part, by grant 1P01GM48874 from the National Institutes of Health, and grant DMS-8920550 from NSF, AFOSR, and ONR.

\section{References}

[1] Emile Aarts, and Jan Korst [1989]. Simulated Annealing and Boltzmann Machines. John Wiley \& Sons, New York, NY.

[2] Charles L. Brooks III, Martin Karplus and B. Montgomery Pettitt [1988]. Proteins: A Theoretical Perspective of Dynamics, Structure, and Thermodynamics. John Wiley \& Sons, New York, NY.

[3] Richard H. Byrd, Elizabeth Eskow, Robert B. Schnabel and Sharon L. Smith [1991]. Parallel Global Optimization: Numerical Methods, Dynamic Scheduling Methods, and Application to Molecular Configuration. Technical Report CU-CS-553-91, Department of Computer Science, University of Colorado at Boulder, Boulder, CO.

[4] Thomas Coleman, David Shalloway and Zhijun Wu [1992]. Isotropic Effective Energy Simulated Annealing Searches for Low Energy Molecular 
Cluster States. Technical Report CTC92TR113, Advanced Computing Research Institute, Cornell University, Ithaca, NY.

[5] J. E. Dennis, Jr. and R. B. Schnabel [1983]. Numerical Methods for Unconstrained Optimization and Nonlinear Equations. Prentice-Hall, Englewood Cliffs, N.J.

[6] Philip E. Gill, Walter Murray and Margaret H. Wright [1981]. Practical Optimization. Academic Press, London.

[7] Bruce A. Hendrickson [1991]. The Molecular Problem: Determining Conformation from Pairwise Distances. Ph.D. Thesis, Department of Computer Science, Cornell University, Ithaca, NY.

[8] Brian E. Hingerty, Samuel Figueroa, Thomas L. Hayden and Suse Broyde [1989]. Prediction of DNA Structure from Sequence: A BuildUp Technique. Biopolymers 28, 1195-1222.

[9] M. R. Hoare [1979]. Structure and Dynamics of Simple Microclusters. Advanced Chemical Physics, 40, 49-135.

[10] S. Kirkpatrick, C. D. Gellat, Jr., and M. P. Vecchi [1983]. Optimization by Simulated Annealing. Science 220, 671-680.

[11] J. Kostrowicki, L. Piela, Binny J. Cherayil and Harold A. Scheraga [1991]. Performance of the Diffusion Equation Method in Searches for Optimum Structures of Clusters of Lennard-Jones Atoms. Journal of Physical Chemistry 95, 4113-4119.

[12] Z. Li and Harold A. Scheraga [1987]. Monte Carlo Approach to the Multiple-Minima Problem in Protein Folding. Proceedings of National Academy of Science, USA, 84, 15-29.

[13] Shang-keng Ma [1973]. Introduction to the Renormalization Group. Reviews of Modern Physics, 45, No. 4, 589-614.

[14] N. Metropolis, A. Rosenbluth, M. Rosenbluth, A. Teller, and E. Teller [1953]. Equation of State Calculations by fast Computing Machines. Journal of Chemical Physics 21, 1087-1092. 
[15] J. A. Northby [1987]. Structure and Binding of Lennard-Jones Clusters: $13 \leq n \leq 147$. Journal of Chemical Physics 87, 6166-6178.

[16] L. Piela, J. Kostrowicki, and Harold A. Scheraga [1989]. The MultipleMinima Problem in the Conformational Analysis of Molecules. Deformation of the Protein Energy Hypersurface by the Diffusion Equation Method. Journal of Physical Chemistry 93, 3339-3346.

[17] M. Pincus, R. Klausner and H. Scheraga [1982]. Calculation of the ThreeDimensional Structure of the Membrane-Bound Portion of Melittin from its Amino Acid Sequence. Proceedings of National Academy of Science, USA, 79, 5107-5110.

[18] David Shalloway [1992]. Packet Annealing: A Deterministic Method for Global Minimization. Application to Molecular Conformation. In C. Floudas and P. Pardalos, eds, Global Optimization, Princeton University Press, Princeton, NJ.

[19] David Shalloway [1992]. Application of the Renormalization Group to Deterministic Global Minimization of Molecular Conformation Energy Functions. Global Optimization 2, 281-311.

[20] Jae Kwang Shin and Mu Shik Jhon [1991]. High Directional Monte Carlo Procedure Coupled with the Temperature Heating and Annealing as a Method to Obtain the Global Energy Minimum Structure of Polypeptides and Proteins. Biopolymers 31,177-185.

[21] David Vanderbilt and Steven G. Louie [1984]. A Monte Carlo Simulated Annealing Approach to Optimization over Continuous Variables. Journal of Computational Physics 59, 259-271.

[22] L. T. Wille [1987]. Minimum-Energy Configuration of Atomic Clusters: New Results Obtained by Simulated Annealing. Chemical Physics Letters 133, 405-410.

[23] K. G. Wilson [1975]. The Renormalization Group: Critical Phenomena and the Kondo Problem. Review of Modern Physics 47, 773-840. 
[24] Guo-liang Xue [1992]. Improvement on the Northby Algorithm for Molecular Conformation: Better Solutions. Army High Performance Computing Research Center, University of Minnesota, MN. 